\title{
Extraction of elastic modulus of porous ultra-thin low-k films by two-dimensional finite- element simulations of nanoindentation
}

O. O. Okudur, K. Vanstreels, I. De Wolf, and U. Hangen

Citation: Journal of Applied Physics 119, 025302 (2016);

View online: https://doi.org/10.1063/1.4939284

View Table of Contents: http://aip.scitation.org/toc/jap/119/2

Published by the American Institute of Physics

\section{Articles you may be interested in}

Thermal expansion coefficients of ultralow-k dielectric films by cube corner indentation tests at elevated temperatures

Applied Physics Letters 107, 233101 (2015); 10.1063/1.4936996

PECVD low and ultralow dielectric constant materials: From invention and research to products Journal of Vacuum Science \& Technology B, Nanotechnology and Microelectronics: Materials, Processing, Measurement, and Phenomena 34, 020801 (2016); 10.1116/1.4943049

$1 / f$ noise measurements for faster evaluation of electromigration in advanced microelectronics interconnections Journal of Applied Physics 119, 184302 (2016); 10.1063/1.4947582

Cu passivation for integration of gap-filling ultralow-k dielectrics

Applied Physics Letters 109, 232901 (2016); 10.1063/1.4971774

Intrinsic effect of porosity on mechanical and fracture properties of nanoporous ultralow-k dielectrics Applied Physics Letters 101, 123109 (2012); 10.1063/1.4753972

Young's Modulus, Shear Modulus, and Poisson's Ratio in Silicon and Germanium Journal of Applied Physics 36, 153 (2004); 10.1063/1.1713863

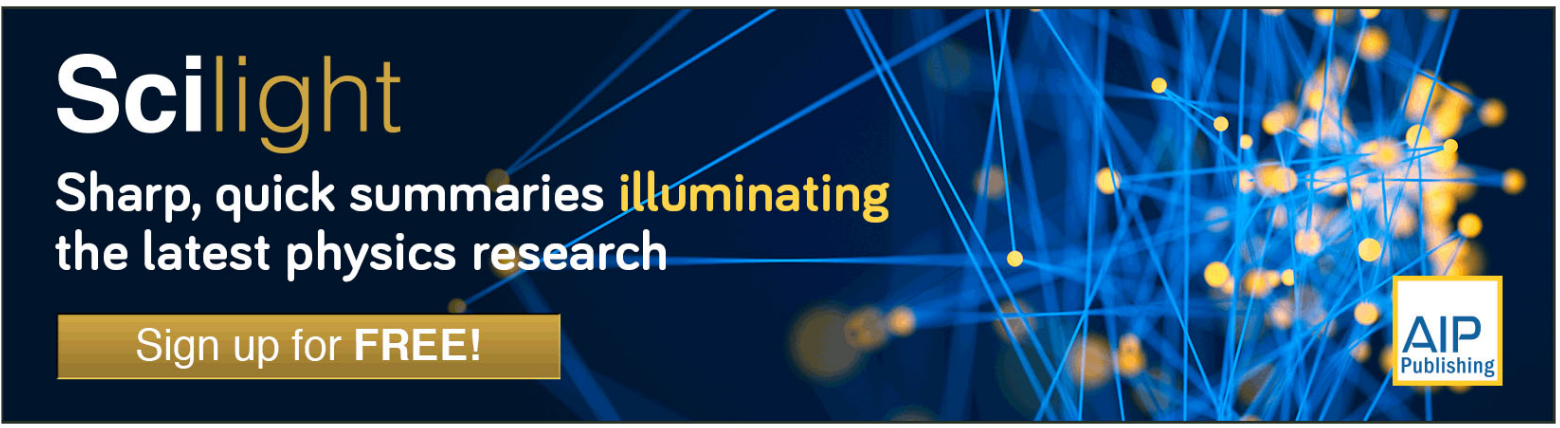




\title{
Extraction of elastic modulus of porous ultra-thin low-k films by two-dimensional finite-element simulations of nanoindentation
}

\author{
O. O. Okudur, ${ }^{1,2, a)}$ K. Vanstreels, ${ }^{2}$ I. De Wolf, ${ }^{1,2}$ and U. Hangen ${ }^{3}$ \\ ${ }^{1}$ Department of Materials Engineering, KU Leuven, B-3000 Leuven, Belgium \\ ${ }^{2}$ IMEC, Kapeldreef 75, B-3001 Leuven, Belgium \\ ${ }^{3}$ Hysitron, Inc., Dennewartstrasse 25, 52068 Aachen, Germany
}

(Received 8 October 2015; accepted 19 December 2015; published online 8 January 2016)

\begin{abstract}
Continuous scaling of integrated circuits has led to the introduction of highly porous low dielectric constant (low-k) materials, whose inferior mechanical properties raise concerns regarding the reliability of integrated circuits. Nanoindentation is proven to be a straightforward method to study mechanical properties of films. However, in the case of low-k, the measurement and analysis are complex due to the porous nature of the films and reduced film thicknesses which give rise to substrate effects. A methodology that combines nanoindentation experiments with finite-element simulations is proposed and validated in this study to extract the substrate-free elastic modulus of porous ultra-thin low-k films. Furthermore, it is shown that imperfections of the nanoindentation probe significantly affect the finite-element results. An effective analytical method that captures the actual nanoindenter behavior upon indentation is proposed by taking both tip radius and conical imperfections into account. Using this method combined with finite element modeling, the elastic modulus of sub-100 nm thick low-k films is successfully extracted. Standard indentation tests clearly overestimated the actual modulus for such thin films, which emphasizes the importance of the proposed methodology. (C) 2016 AIP Publishing LLC. [http://dx.doi.org/10.1063/1.4939284]
\end{abstract}

\section{INTRODUCTION}

The continuous scaling of integrated circuits (ICs) necessitated the replacement of conventionally used $\mathrm{SiO}_{2}$ by porous ultra low dielectric constant materials (ultra low-k) to compensate for problems exacerbated by the scaling, such as RC delay, cross talk, power consumption, and noise. ${ }^{1}$ However, the introduction of these materials into ICs has been a difficult task so far, due to a number of integration and reliability issues. ${ }^{2}$ A considerable amount of these issues are associated with the inferior mechanical properties of the ultra-low-k materials. ${ }^{3}$ On top of that, since these materials exhibit intrinsic tensile stresses and have higher coefficients of thermal expansion compared with $\mathrm{SiO}_{2}$, thin film cracking and adhesion are serious thermo-mechanical reliability issues. ${ }^{4}$ Therefore, it is important to assess the mechanical properties of these films in a reliable and reproducible way. To date, nanoindentation is the most commonly used technique in the semiconductor industry to assess the mechanical properties of thin low-k films such as elastic modulus and hardness. ${ }^{3}$ Although the measurement of elastic properties with nanoindentation seems almost a routine job, the analysis is rather complex due to the porous nature of the films and the reduced film thicknesses $(<100 \mathrm{~nm})$ that are used in advanced technology nodes. Recently, Vanstreels et al. demonstrated a clear correlation between the internal pore structure of ultra low-k films and their mechanical response upon indentation, thereby improving the fundamental understanding on how the measured elastic modulus value is influenced by porosity, the pore structure, the matrix stiffness, and

\footnotetext{
a) Author to whom correspondence should be addressed. Electronic mail: oguzhan.orkut.okudur@imec.be
}

material/probe interactions. ${ }^{5}$ On the other hand, low-k films with reduced thicknesses cause the stiffness of the substrate to interfere with the measured elastic modulus, thus leading to the overestimation or underestimation of the actual elastic modulus. ${ }^{5}$ This phenomena is evident in all type of thin films deposited on a substrate and referred to as the substrate effect in the literature. ${ }^{6,7} \mathrm{~A}$ rule of thumb states that in order to avoid severe substrate effects, the penetration depth should be less than $10 \%$ of the film thickness. This is certainly not a universal law, but requires good knowledge of the indentation behavior of the film/substrate system. A commonly used procedure to minimize the substrate effect is by reducing the spatial extent of the elastic-deformation field upon indentation. This can be achieved by using a cube corner probe instead of the standard Berkovich three-sided pyramid probe. $^{8}$ Another approach is by reducing the indentation depth at which the elastic modulus is extracted, typically below $15 \mathrm{~nm}$. However, the analysis is still often affected by the substrate when the films are ultra-thin. The Oliver-Pharr model $^{9}$ is the most widely accepted model to analyze the nanoindentation data. However, this model is applicable for bulk materials and no longer sufficient to accurately determine the elastic modulus when there is substrate effect. A number of analytical models to remove the substrate effect by modifying the Oliver-Pharr approach were presented over the last decades. ${ }^{6,10-14}$ A recent review by $\mathrm{Li}$ and co-authors compared these models in terms of their weaknesses and strengths. ${ }^{15}$ Finite element modelling (FEM) is an alternative approach for direct extraction of the substrate independent modulus and has been extensively used in conjunction with nanoindentation experiments to investigate the substrate effect. $^{5,16-18}$ However, this approach can only result in 
meaningful values for the elastic modulus if the indenter tip shape can be accurately represented in the simulations.

The purpose of this paper was to present a new methodology to obtain substrate independent mechanical properties of porous ultra-thin low-k films $(<100 \mathrm{~nm})$ by combining experimental studies and FEM. To this end, it is necessary to accurately simulate the nanoindenter geometry, including imperfections. This is achieved by a shape area fitting approach based on experimental calibration data.

\section{NANOINDENTER GEOMETRY}

The finite element model for nanoindentation simulations can either be built based on a pyramidal geometry in 3D (Figure 1(a)) or on a conical geometry in 2D (Figure 1(b)). 2D axisymmetric FEM provides decreased complexity and computational cost, and is usually preferred whenever possible. It was shown that pyramidal indenters such as Berkovich and cube corner can be successfully modeled in 2D using equivalent cone geometries that will provide the same contact depth to contact area relationship. ${ }^{18,19}$ The contact area $\left(\mathrm{A}_{\mathrm{c}}\right)$ of an ideal pyramidal tip with centerline to face angle $\alpha$ (Figure 1(a)) and an ideal conical indenter with angle $\theta$ (Figure 1(b)) can be calculated as a function of contact depth $\left(\mathrm{h}_{\mathrm{c}}\right)$ using Eqs. (1) and (2), respectively. From these equations, equivalent cone angles to represent Berkovich $\left(\alpha=65.3^{\circ}\right)$ and cube corner $\left(\alpha=35.3^{\circ}\right)$ indenters in $2 \mathrm{D}$ are found to be $70.3^{\circ}$ and $42.3^{\circ}$, respectively. This is done by calculating $\mathrm{A}_{\mathrm{c}}$ from Eq. (1) using the known value of $\alpha$ and extracting $\theta$ from Eq. (2).

$$
\begin{gathered}
A_{c}=3 \sqrt{3} h_{c}^{2} \tan ^{2} \alpha, \\
A_{c}=\pi h_{c}^{2} \tan ^{2} \theta .
\end{gathered}
$$

However, these equations do not take into account the deviations of the tip from ideality. From the experimental point of view, a well adopted methodology to calibrate the contact area in nanoindentation measurements is to use the method developed by Oliver-Pharr, ${ }^{20}$ where series of indentations at different contact depths are performed on a sample with known elastic modulus. Contact areas at different depths are then derived using

$$
S=\beta \frac{2}{\sqrt{\pi}} E_{r} \sqrt{A_{c}},
$$

where $\beta$ is a constant which depends on the geometry of the indenter, $\mathrm{E}_{\mathrm{r}}$ is the reduced elastic modulus, and the stiffness (S) is calculated from the initial portion of the unloading curve. Following the calculation of contact area at different depths, a function to correlate the contact area to contact depth is fitted based on the following formula. ${ }^{20}$
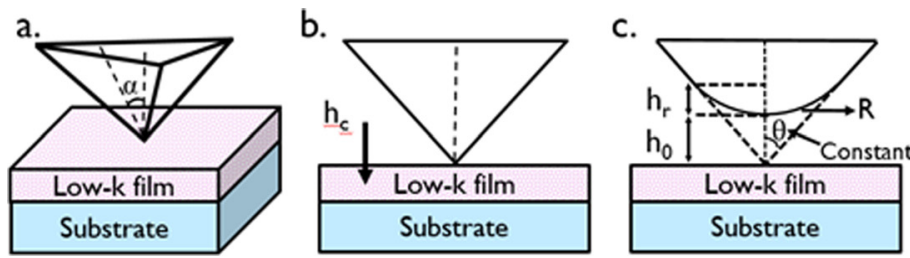

d.

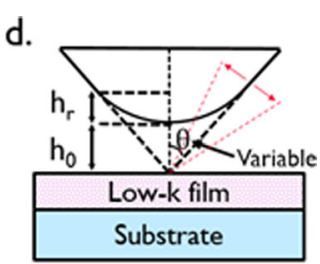

$$
A_{c}=C_{o} h_{c}^{2}+C_{1} h_{c}+C_{2} h_{c}^{1 / 2}+C_{3} h_{c}^{1 / 4}+C_{4} h_{c}^{1 / 8}+\cdots .
$$

In case of an ideal indenter probe (Figs. 1(a) and 1(b)), the contact area (Eqs. (1) and (2)) can solely be represented by the first term $\left(\mathrm{C}_{\mathrm{o}} \mathrm{h}_{\mathrm{c}}{ }^{2}\right)$ in Eq. (4), where $\mathrm{C}_{\mathrm{o}}$ can be derived from either Eqs. (1) or (2) which yield the values 24.5 and 2.6 for ideal Berkovich and cube corner indenters, respectively. In reality, this is almost never the case, since all tips have some degree of imperfection. The additional empirical terms in Eq. (4) compensate these deviations from ideality. The most recognized tip imperfection for pyramidal indenters is the tip radius, $\mathrm{R}$ (Figure 1(c)), which can vary according to the quality of production and wearing of the tip throughout the usage. If one considers the fact that indentations are typically performed at thicknesses below $15 \mathrm{~nm}$ for ultra-thin films, most of the actual indentation process, if not all, will be performed by those imperfections at the end of the tip. Therefore, it is apparent that it would lead to wrong conclusions when the indenter geometries that are used in FEM fail to incorporate the deviations from ideality. Hence, it is crucial to determine the representative tip shape for FEM that would properly represent the behavior of the tip upon indentation. ${ }^{21}$ Although direct measurement techniques such as SEM and AFM can be applied to determine the tip geometry, ${ }^{22-24}$ they are usually difficult and take quite some time. Furthermore, the continuous wearing of the tip throughout the usage makes analytical methods more practical since these methods do not necessitate any additional experiments. In fact, the experimental contact depth-contact area calibration curve is all that is needed and should already be available when the Oliver-Pharr method is being used to analyze the data. Some useful analytical methods based on physical interpretations of the indenter geometries and associated imperfections have been reported to determine the tip geometry. These methods are based on fitting the experimentally obtained calibration data by spherical, conical, and/or spherical-conical functions. ${ }^{17,23,25-29}$

One of the most widely used methods to determine the tip radius is to fit the experimental contact area to contact depth points by the spherical area $\left(\mathrm{A}_{\text {spherical }}\right)$ function, given in Eq. (5), which allows one to extract the tip radius, $R$

$$
A_{\text {spherical }}=\pi h_{c}\left(2 R-h_{c}\right) .
$$

The main drawback of this method is that it only considers the radial part and does not provide any information regarding the conical part of the indenter. Another disadvantage is that the range of contact depth selected for fitting has a high influence on the extracted tip radius. Moreover, determining the exact transition point at which indentation becomes conical rather than spherical brings another ambiguity. This analytical method was further improved by implementing the

FIG. 1. Different geometrical representations for pyramidal nanoindenter probe: (a) ideal pyramidal indenter, (b) equivalent ideal conical indenter, (c) ideal conical indenter with a spherical extremity, and (d) proposed geometry for $2 \mathrm{D}$ simulations. 
conical area function with a spherical extremity to the fitting procedure (Figure 1(c)). It was shown that the tip radius, R, can be calculated by simultaneous least-squares fitting of the two nonlinear equations given in Eqs. (6) and (7) to extract the value of $\mathrm{R}^{17}$

$$
\begin{gathered}
A_{\text {spherical }}=\pi h_{c}\left(2 R-h_{c}\right), \quad \text { for } h_{c} \leq h_{r}, \\
A_{\text {conical }}=\pi \tan ^{2} \theta\left(h_{c}+h_{0}\right)^{2}, \quad \text { for } h_{c}>h_{r},
\end{gathered}
$$

where $h_{r}$ is the contact depth of penetration performed by the radial part of the tip which can be considered as the contact depth of transition from spherical to conical penetration and $\mathrm{h}_{0}$ is the distance between the apex of the actual indenter and the apex of the ideal conical indenter as shown in Figure 1(c). Both $h_{r}$ and $h_{0}$ are dependent on the tip radius and their values can be calculated by using Eqs. (8) and (9), respectively,

$$
\begin{gathered}
h_{r}=R-R \sin \theta, \\
h_{0}=R / \sin \theta-R .
\end{gathered}
$$

This fitting procedure takes into account both tip radius and the conical part of the indenter. However, the conical part of the indenter is assumed to be ideal $\left(\pi \tan ^{2} \theta\right.$ is a fixed constant for each tip). This assumption may not always represent the reality since the conical part is a representation of the pyramidal part which can have some deviations from the ideality in terms of pyramidal angle, wall radius, asymmetry, and/ or alignment. Failing to consider the cone imperfections may result in extracting a tip radius that is not representative of the actual indentation process. Therefore, it is necessary to include another variable that can compensate the effects other than the tip radius. It is proposed in this study that the spherical-conical fitting method may further be enhanced by considering the deviations caused by the conical part as well. To this end, the value of parameter $\mathrm{C}_{\mathrm{o}}$ (Eq. (12)), which was experimentally already found to give different values for different tips, ${ }^{29-31}$ is in our approach not kept constant but taken as an additional variable together with the tip radius (R). The constant $\theta$ is thus replaced by the variable $C_{0}$. The governing equations of the spherical-conical fitting then become

$$
\begin{gathered}
A_{\text {spherical }}=\pi h_{c}\left(2 R-h_{c}\right), \quad \text { for } h_{c} \leq h_{r}, \\
A_{\text {conical }}=C_{0}\left(h_{c}+R / \sin \left(\arctan \left(\sqrt{C_{0} / \pi}\right)\right)-R\right)^{2}, \\
\text { for } h_{c}>h_{r}, \\
C_{0}=\pi \tan ^{2} \theta .
\end{gathered}
$$

Simultaneous nonlinear least-squares fitting of Eqs. (10) and (11) with $\mathrm{R}$ and $\mathrm{C}_{\mathrm{o}}$ as the regression parameters allows to derive the necessary parameters to simulate any pyramidal indenter with a certain angle. It should be noted that the form of spherical-conical fitting provided in Eqs. (10) and (11) would practically be the same with Eqs. (8) and (9) when $\theta$ is considered as a variable rather than a constant. The former, however, allows one to calculate conical area function $\left(\mathrm{C}_{0}\right)$ and radius $(\mathrm{R})$ together and directly observe the deviations due to the conical and spherical imperfections. From this, the value of $\theta$ for 2D and $\alpha$ for 3D simulations can indirectly be calculated and used. In either case, the experimental data are fitted with two area functions that capture possible imperfections of both spherical/conical parts. The change in $\mathrm{C}_{0}$ can be transferred to $2 \mathrm{D}$ FEM simulations by varying $\theta$ as shown in Figure 1(d).

Bei et al. indicated that although it is a reasonable first approach, the tip of Berkovich indenters are not really spherical. Instead, they should rather be represented by a more complicated geometry. ${ }^{32}$ It was suggested to fit the contact area by Eq. (13). This equation has a simple physical interpretation. While the first term represents a conical indenter, the second term describes a spherical indenter. It provides a smooth transition between two limiting cases of spherical contact at low depths and conical contact above the transition point

$$
A=\alpha_{1} h_{c}^{2}+\alpha_{2} h_{c}
$$

So far, different contact area fitting procedures are described including ideal shape assumption (Fig. 1(b)), spherical fitting (Fig. 1(c)), spherical-perfect conical fitting (Fig. 1(c)), the proposed fitting procedure (Fig. 1(d)), and the model of Bei et $a l .^{32}$ These five different fitting procedures are compared using Berkovich and cube corner indenters. Results of the fitting processes are shown in Figures 2 and 3. Experimental data points were obtained by making several indents on a reference fused silica sample at different indentation depths using a Hysitron TI950 triboindenter. Table I provides a summary of the extracted $\mathrm{R}, \mathrm{C}_{0}$, and consequently $\theta$ values extracted from the different fitting processes for the Berkovich tip.

As can be seen from Figures 1 and 2, fitting performed using the proposed method agrees very well with the experimental data and is superior to the other methods except for the method proposed by Bei et al., for both indenter tips. This indicates that the new indenter model created based on the extracted $\mathrm{C}_{0}$ and $\mathrm{R}$ values can properly represent the contact area as a function of contact depth which is the information required to do correct finite element simulations. If this is the case, load-displacement data extracted from an FE

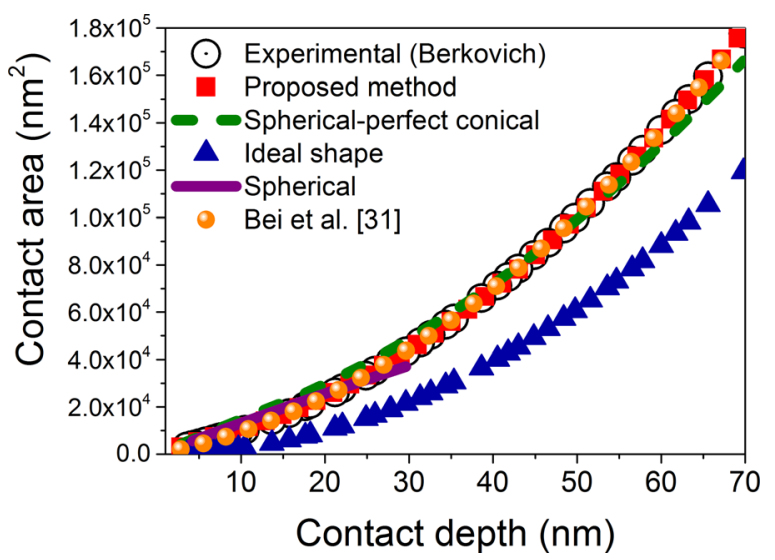

FIG. 2. Fitting of the experimental contact area calibration curve for a Berkovich tip with different geometrical considerations. 


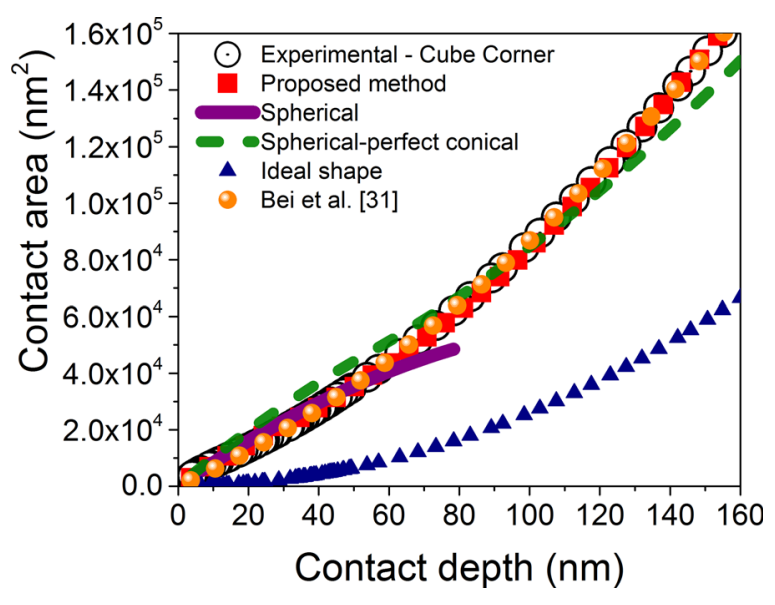

FIG. 3. Fitting of the experimental contact area calibration curve for a cube corner tip with different geometrical considerations.

model should be comparable with the actual indentation process observed during the nanoindentation experiments.

\section{RESULTS AND DISCUSSION}

\section{A. Reference sample (fused silica)}

To test this assumption and methodology, 2D axisymmetric finite-element simulations of Berkovich indentation were performed using the commercial finite-element code MSC.MARC. Indenter geometries were modeled using the derived $\mathrm{R}$ and $\theta$ values. Five different $\mathrm{FE}$ models were created to test the correctness of the five different indenter geometries extracted using different fitting methodologies as outlined in Table I. The summary of the experimental and modeling process along with the mesh/indenter configurations are shown in Figure 4.

The indenter probes were modeled as axisymmetric cones with a certain radius at the tip. They were considered as rigid bodies and constrained to move only in the direction of the indentation with a displacement control. The mesh density at the contact region between the indenter and the sample was increased to ensure the simulation accuracy. Fused silica was used as a first sample to test the methodology and modeled as an elastic-plastic solid. The elastic modulus of fused silica was derived from Eq. (14), where $\nu$ is the Poisson's ratio, and subscripts $i$ and $s$ refer to the indenter and sample (fused silica), respectively.

$$
\frac{1}{E_{r}}=\frac{1-v_{i}^{2}}{E_{i}}+\frac{1-v_{s}^{2}}{E_{s}} .
$$

Using the experimentally measured reduced modulus $\left(\mathrm{E}_{\mathrm{r}}\right)$ of 69.6 GPa and considering a rigid indenter model (first term becomes zero), $\mathrm{E}_{\mathrm{s}}$ is found to be $67.6 \mathrm{GPa}$. The yielding behavior was modeled by the Drucker-Prager yield criterion with a yield strength of $5.5 \mathrm{GPa}^{33}$

Results of the experiments performed on fused silica together with the FE simulations with the different indentershape models from Table I are given in Figure 5. As was expected, the ability of a particular fitting method to follow the contact depth-contact area relationship of the sample (Figure 2) is reflected in its ability to simulate the actual indentation process during FE simulations. The FE model built based on the ideal shape is found to be far away from the experimental load-displacement characteristics. This is an expected result since this method does not consider any imperfection of the tip. In the case of spherical fitting, the loading curve is reproduced in a quite reasonable manner up to a certain depth above which the conical part of the indenter dominates and the model starts deviating from the data. Surprisingly, the spherical-perfect conical fitting method could not reproduce the loading curve as well as the spherical fitting method, although they use the same function, given in Eq. (5), to represent the behaviour of indentation in the spherical regime. However, assuming the conical shape as perfect when it was not, affected the value of the extracted radius, thus resulted in inaccurate modeling. It should be noted that all these fitting methods are based on certain assumptions such as ideal shape, certain radius but perfect conical geometry, etc. It is also a fact that the degree and type of imperfection is unique for each and every tip. Therefore, depending on how well these assumptions represent the reality, success of different fitting methods may be different for different tips. The proposed fitting method provides an almost complete match with the experimental results in terms of retracing the load-displacement characteristics as was expected because it provides more flexibility to account for possible imperfections for the radial and conical parts as well as alignment issues. Load-displacement data obtained from the FE model performed with the indenter geometry derived from the fitting model of Bei et al. are found to poorly follow the experimental characteristics. At a first glance, this is surprising since the contact area to contact depth can successfully be fitted with Eq. (13) as shown in Figures 2 and 3. However, this fitting process does not use separate equations to describe the indenter at spherical and conical regimes, rather it suggests to use a single equation where the terms are summed up. Therefore, the data transferred to 2D FEM by spherical-conical indenter geometry cannot truly represent the behavior of the nanoindenter. Nevertheless, it was not the intention of the authors to use the fitting process for 2D FEM simulations. What we aim to find was a fitting method that can derive an equivalent (effective) nanoindenter geometry that directly can be used for 2D FEM, even though the extracted parameters do not

TABLE I. Berkovich indenter geometries to be used at FEM, derived by different fitting methods.

\begin{tabular}{|c|c|c|c|c|c|}
\hline Extracted parameters & Ideal shape & Spherical fitting & Spherical-perfect conical fitting & Proposed method & Bei et al. ${ }^{32}$ \\
\hline $\mathrm{R}(\mathrm{nm})$ & 0 & 214 & 250 & 150 & 109 \\
\hline $\mathrm{C}_{0}$ & 24.5 & 24.5 & 24.5 & 38 & 26.6 \\
\hline$\theta\left({ }^{\circ}\right)$ & 70.3 & 70.3 & 70.3 & 74 & 71 \\
\hline
\end{tabular}




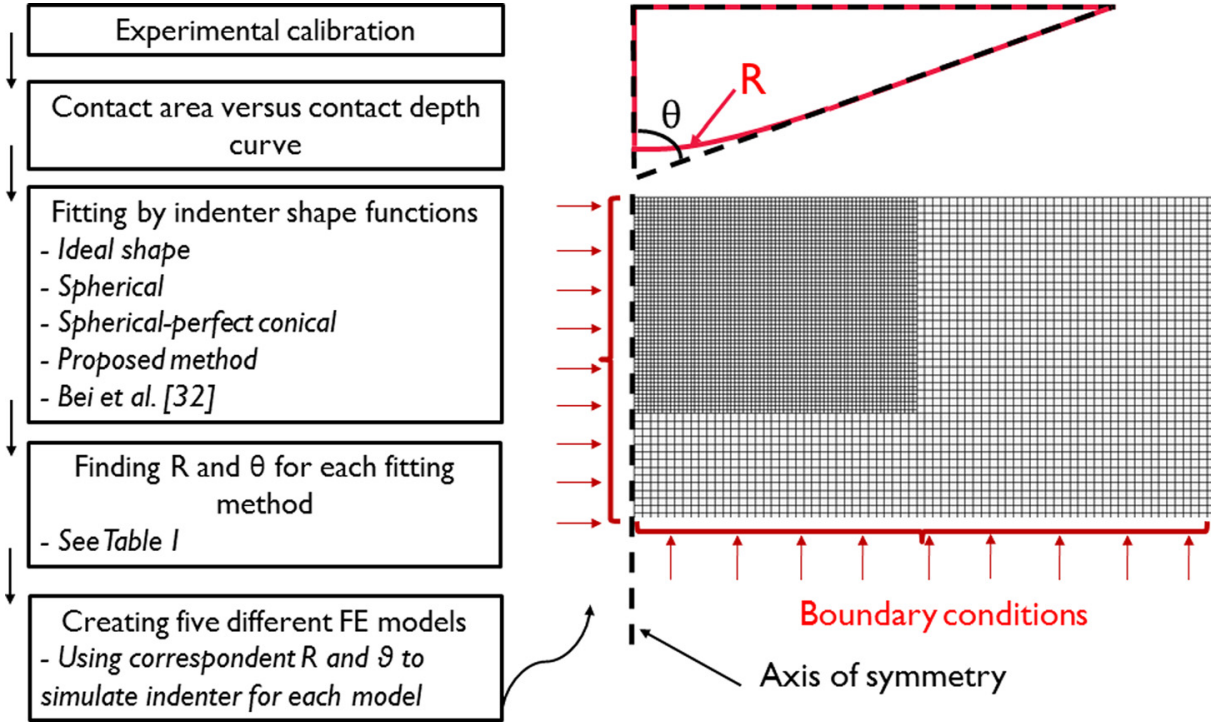

FIG. 4. Summary of the experimental flowchart and FEM of indentation process. necessarily represent the accurate and true geometry of the indenter. The proposed fitting method yields the most promising results for this purpose.

\section{B. Low-k films}

\section{Berkovich indentation}

Upon successful confirmation of the simulated indenter shape derived by the proposed fitting method, further experiments with the Berkovich tip were conducted using porous thin low-k films deposited on a silicon substrate. It should be noted that both FEM and experiments are performed at ultrashallow depths in this technique; therefore, the accuracy of the technique may not be as good if the surface is too rough due to the variations of the actual contact area and the substrate effect. Therefore, all the low-k films used throughout this study had smooth surface finishes (sub nm roughness). The elastic modulus of two low-k films, deposited by plasma enhanced chemical vapor deposition (PECVD) to the thicknesses of $235 \mathrm{~nm}$ and $191 \mathrm{~nm}$ with porosity levels around

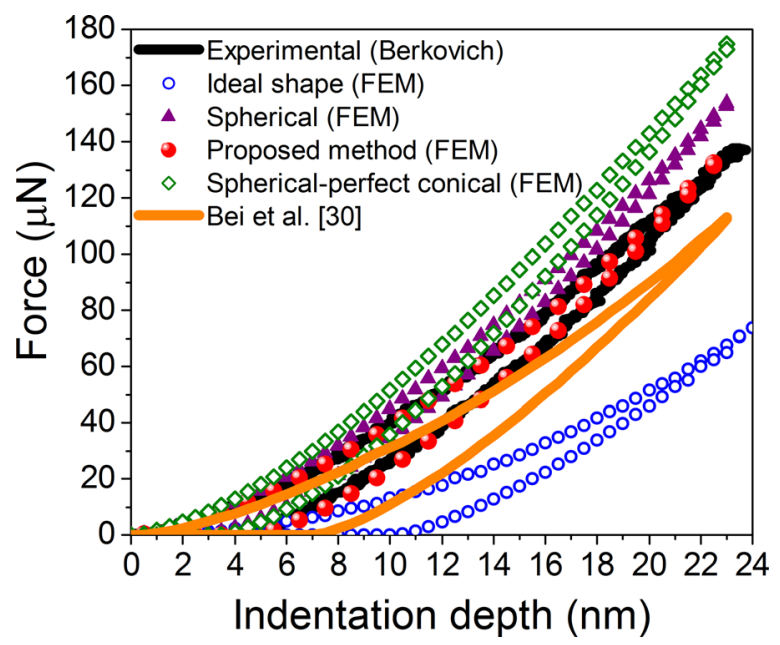

FIG. 5. Force-indentation depth data of fused silica obtained from experiments and finite-element simulations performed with different indenter geometries derived from different fitting methods.
$24 \%$ and $38 \%$, were measured experimentally. FE simulations were performed using the derived indenter shape from the proposed fitting process and the experimentally measured elastic modulus value as input. These results, together with the data from the reference fused silica sample, are presented in Figure 6. As shown, simulations performed at sub- $15 \mathrm{~nm}$ thicknesses were found to very accurately follow the experimental load-displacement characteristics for different materials, thicknesses, and depths.

\section{Cube corner indentation}

To even further validate the methodology, another indenter probe with a different geometrical configuration was tested. For this purpose, a cube corner indenter was used and modeled with a $128 \mathrm{~nm}$ tip radius and the $\mathrm{C}_{0}$ value of 4.23 as extracted from the fitting process shown in Figure 2. The elastic modulus of two low-k films, deposited by PECVD and spin-on method to the thicknesses of $235 \mathrm{~nm}$ and $201 \mathrm{~nm}$ with porosity levels around $24 \%$ and $34 \%$, respectively, were measured experimentally at sub-10 nm thicknesses and

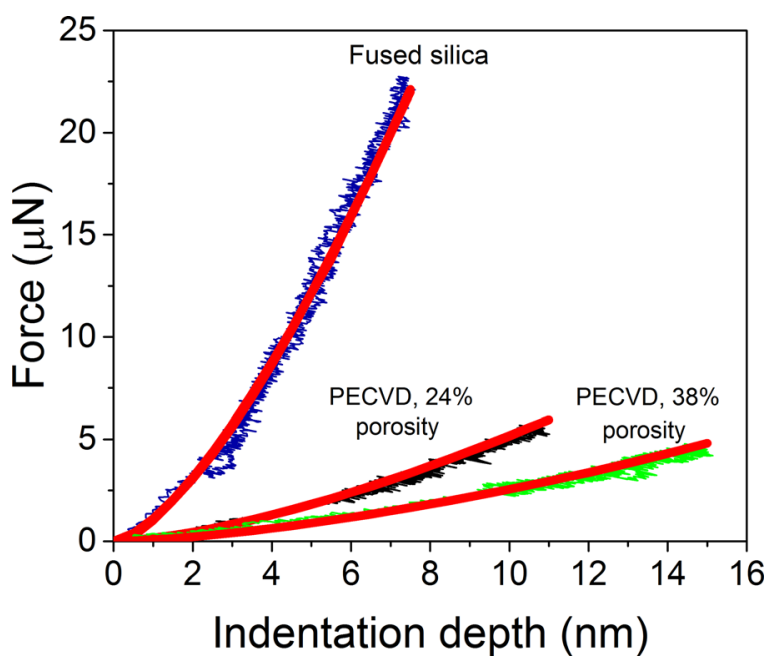

FIG. 6. Comparison of force-indentation depth data obtained from FEM (red lines) and experiments for Berkovich indentation. 
results were compared with FEM (Figure 7). Similar to the results observed for the Berkovich tip, the FE model performed using the derived cube corner indenter shape by the fitting process, was very successful to simulate the experimental data. This shows that the methodology can be applied to different types of pyramidal indenters, regardless of the pyramidal angle.

\section{Ultra-thin low-k films}

So far, the discussions were limited to samples whose mechanical property measurements are not severely affected by the substrate. The results indicate that, when the nanoindentation probe is modeled using the proposed fitting methodology, it becomes possible to accurately simulate experimental load-displacement curves even at very shallow depths. To demonstrate this for thinner films, the same methodology was applied to organo-silicate-glass (OSG) type porous ultra-thin low-k films which were prepared to have similar properties but different thicknesses $(43 \mathrm{~nm}, 86 \mathrm{~nm}$, and $310 \mathrm{~nm}$ on silicon substrate). Elastic modulus (E) values of these three types of films were expected to be close to each other, possibly slightly differing due to small processing variations. Experiments were performed with a cube corner tip below $15 \mathrm{~nm}$ indentation depth. The experimentally obtained E-values are shown in Table II. Values given in the table represent the average and standard deviation (s) of experiments for each film. Standard deviations are found to be higher than usual due to the very low displacements used in the tests. While the true elastic modulus of the film with $310 \mathrm{~nm}$ thickness could be obtained without the substrate effect owing to indentation depth being smaller than $5 \%$ of the total film thickness, the measured elastic modulus for films with thicknesses $43 \mathrm{~nm}$ and $86 \mathrm{~nm}$ were found to be drastically increasing as the thickness of the film decreases which clearly demonstrates the overestimation of the elastic modulus due to the substrate effect. To derive the correct $\mathrm{E}$ value, FE simulations using the derived effective indenter geometry were performed by iterating the elastic modulus of the low-k film until the force-displacement curves obtained by the experiments could be retraced by the FEM. The range

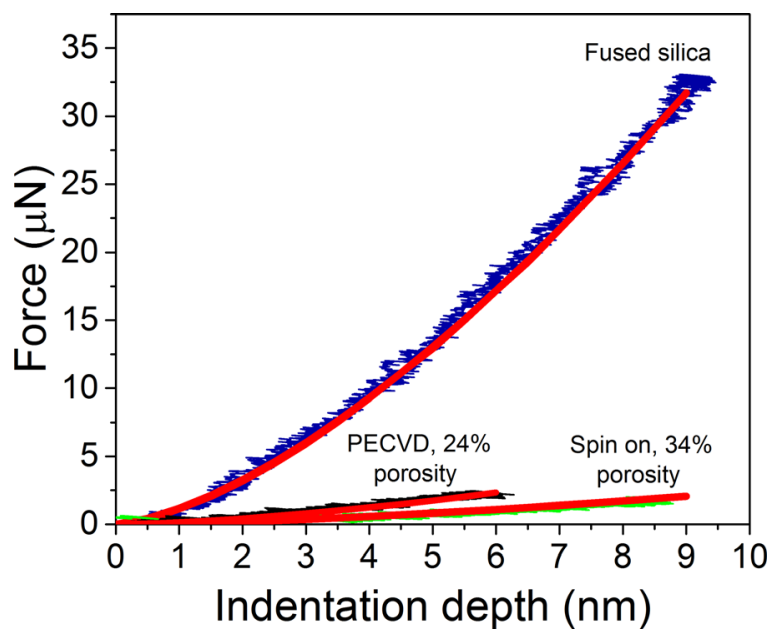

FIG. 7. Comparison of force-indentation depth data obtained from FEM (red lines) and experiments for cube corner indentation.
TABLE II. Extracted elastic modulus values by experimental studies and FEM for OSG 2.4 low-k films with different thicknesses.

\begin{tabular}{lccc}
\hline \hline Thin film & OSG 2.4 & OSG 2.4 & OSG 2.4 \\
\hline Thickness (nm) & 310 & 86 & 43 \\
E-experimental $(\mathrm{GPa})$ & $5.06(\mathrm{~s}=0.67)$ & $9.69(\mathrm{~s}=0.45)$ & $15.60(\mathrm{~s}=1.95)$ \\
E-FEM $(\mathrm{GPa})$ & $5.16(\mathrm{~s}=0.38)$ & $5.76(\mathrm{~s}=0.51)$ & $5.90(\mathrm{~s}=0.40)$ \\
\hline \hline
\end{tabular}

of iterations can be determined by performing a few screening runs starting with the experimentally measured value and lowering it down to more reasonable values. The process can also be automated using specific algorithms and feedback loops with an optimization software such as Optimus, to find the modulus value that minimizes the residual sum of squares of the experimental and finite-element analysis force-displacement curves. For this case, the experimentally measured E-modulus of the $310 \mathrm{~nm}$ thick film $(5.06 \mathrm{GPa})$ was used as the starting point for iterations. Figure 8 presents an example for the experimental data points and corresponding results for FE simulations for films with different thicknesses. The elastic modulus values obtained from FEM are also reported in Table II. For each film, five different experimental load-displacement curves were used, separately iterated to find the best fitted elastic modulus in FEM and standard deviations were calculated accordingly. The extracted values can be considered to be quite reasonable since the experimentally measured elastic modulus of the $310 \mathrm{~nm}$ thick film, where substrate effects have negligible influence, was found to be $5.06 \mathrm{GPa}$ with a standard deviation of 0.67 .

It should be noted that experiments on $43 \mathrm{~nm}$ and $86 \mathrm{~nm}$ thick OSG 2.4 low-k films were performed at different times, $t_{0}$ and $t_{1}$, respectively. It is a well-known fact that the indenter tip may have been subjected to wear throughout its lifetime. Therefore, the fitting methodology was applied separately and different effective nanoindenter geometries were extracted for the same tip at different times as is shown in Figure 9. The fact that the extracted load-displacement

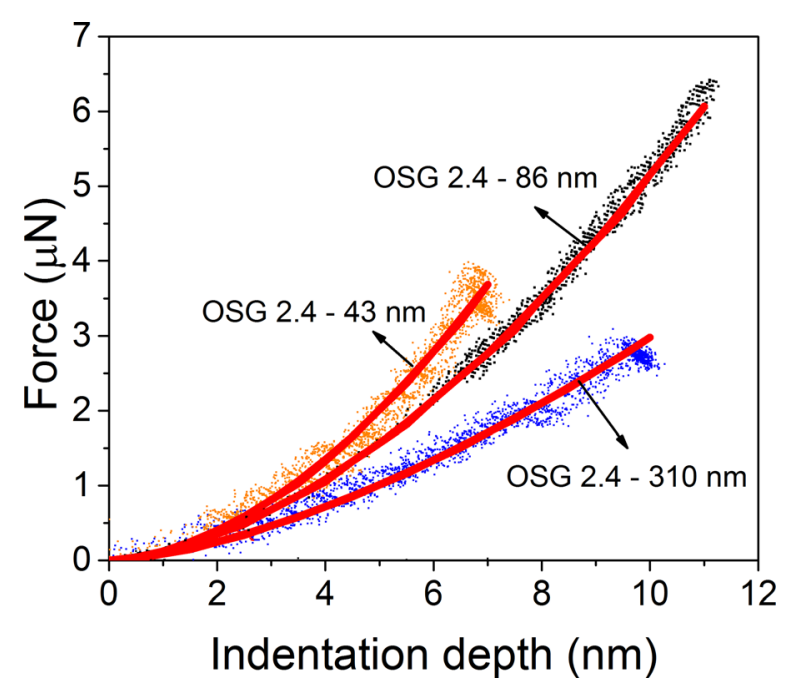

FIG. 8. Experimental data points and corresponding results for FE simulations (red lines) with best fitted elastic modulus values for films with different thicknesses. 


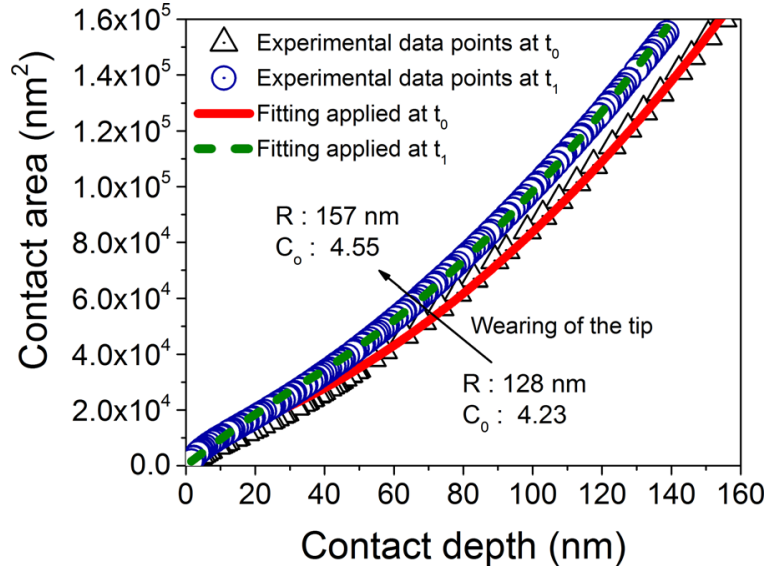

FIG. 9. Fitting of the contact area to contact depth experimental curve of a cube corner indenter by the proposed fitting methodology at different times.

characteristics and the elastic modulus are quite successful for both cases shows that the FEM built based on the proposed fitting methodology is also able to capture the tip wear and/or alignment effects.

\section{CONCLUSIONS}

To conclude, a new methodology that combines nanoindentation experiments with FEM was developed to extract the elastic modulus of porous ultra-thin low- $k$ films. This was done by proper calibration and subsequent simulation of the effective nanoindenter geometry. The validity of the method was proven for two different tips and seven different materials. Moreover, the methodology was also able to capture the effects caused by the wearing of the tip throughout its lifetime.

${ }^{1}$ S.-P. Jeng, M.-C. Chang, T. Kroger, P. McAnally, and R. H. Havemann, "A planarized multilevel interconnect scheme with embedded low-dielectric-constant polymers for sub-quarter-micron applications," in 1994 Symposium on VLSI Technology, Digest of Technical Papers (1994).

${ }^{2}$ K. Maex, M. Baklanov, D. Shamiryan, S. Brongersma, and Z. Yanovitskaya, "Low dielectric constant materials for microelectronics," J. Appl. Phys. 93(11), 8793-8841 (2003).

${ }^{3} \mathrm{~K}$. Vanstreels, C. Wu, and M. Baklanov, "Mechanical stability of porous low-k dielectrics," ECS J. Solid State Sci. Technol. 4(1), N3058-N3064 (2015).

${ }^{4}$ F. Iacopi, S. H. Brongersma, B. Vandevelde, M. O’Toole, D. Degryse, Y. Travaly, and K. Maex, "Challenges for structural stability of ultra-low-kbased interconnects," Microelectron. Eng. 75(1), 54-62 (2004).

${ }^{5}$ K. Vanstreels, C. Wu, M. Gonzalez, D. Schneider, D. Gidley, P. Verdonck, and M. R. Baklanov, "Effect of pore structure of nanometer scale porous films on the measured elastic modulus," Langmuir 29(38), 12025-12035 (2013).

${ }^{6}$ R. Saha and W. D. Nix, "Effects of the substrate on the determination of thin film mechanical properties by nanoindentation," Acta Mater. 50(1), 23-38 (2002).

${ }^{7}$ T. Tsui and G. Pharr, "Substrate effects on nanoindentation mechanical property measurement of soft films on hard substrates," J. Mater. Res. 14(01), 292-301 (1999).

${ }^{8} \mathrm{~K}$. Vanstreels and A. Urbanowicz, "Nanoindentation study of thin plasma enhanced chemical vapor deposition $\mathrm{SiCOH}$ low-k films modified in $\mathrm{He} /$ H-2 downstream plasma,” J. Vac. Sci. Technol. B 28(1), 173-179 (2010).

${ }^{9}$ G. Pharr, W. Oliver, and F. Brotzen, "On the generality of the relationship among contact stiffness, contact area, and elastic modulus during indentation,” J. Mater. Res. 7(03), 613-617 (1992).
${ }^{10} \mathrm{G}$. Huajian, C. Cheng-Hsin, and L. Jin, "Elastic contact versus indentation modeling of multi-layered materials," Int. J. Solids Struct. 29(20), 2471-2492 (1992).

${ }^{11}$ J. Menčík, D. Munz, E. Quandt, E. Weppelmann, and M. Swain, "Determination of elastic modulus of thin layers using nanoindentation," J. Mater. Res. 12(09), 2475-2484 (1997).

${ }^{12} \mathrm{H}$. Li and J. J. Vlassak, "Determining the elastic modulus and hardness of an ultra-thin film on a substrate using nanoindentation," J. Mater. Res. 24(03), 1114-1126 (2009).

${ }^{13}$ Z. Wei, G. Zhang, H. Chen, J. Luo, R. Liu, and S. Guo, "A simple method for evaluating elastic modulus of thin films by nanoindentation," J. Mater. Res. 24(03), 801-815 (2009).

${ }^{14}$ J. Hay and B. Crawford, "Measuring substrate-independent modulus of thin films," J. Mater. Res. 26(06), 727-738 (2011).

${ }^{15}$ Y. Li, P. Valavala, S. Watcharotone, and L. C. Brinson, "Models for nanoindentation of compliant films on stiff substrates," J. Mater. Res. 30(11), $1747-1760$ (2015).

${ }^{16}$ M. Gonzalez, K. Vanstreels, and A. M. Urbanowicz, "Modeling the substrate effects on nanoindentation mechanical property measurement," in 10th International Conference on Thermal, Mechanical and Multi-Physics simulation and Experiments in Microelectronics and Microsystems, EuroSimE 2009 (IEEE, 2009).

${ }^{17}$ N. Yu, A. A. Polycarpou, and T. F. Conry, "Tip-radius effect in finite element modeling of sub-50 nm shallow nanoindentation," Thin Solid Films 450(2), 295-303 (2004).

${ }^{18} \mathrm{~S}$. Liu, H. Huang, and Y. Gu, "Deconvolution of mechanical properties of thin films from nanoindentation measurement via finite element optimization," Thin Solid Films 526(0), 183-190 (2012).

${ }^{19}$ M. Lichinchi, C. Lenardi, J. Haupt, and R. Vitali, "Simulation of Berkovich nanoindentation experiments on thin films using finite element method," Thin Solid Films 312(1-2), 240-248 (1998).

${ }^{20}$ W. C. Oliver and G. M. Pharr, "An improved technique for determining hardness and elastic modulus using load and displacement sensing indentation experiments," J. Mater. Res. 7(06), 1564-1583 (1992).

${ }^{21}$ Y.-H. Lee, Y.-I. Kim, and J.-H. Hahn, "Hardness variation with indenter sharpness in an Au thin-film," J. Nanosci. Nanotechnol. 12(7), 5648-5652 (2012).

${ }^{22}$ L. Calabri, N. Pugno, C. Menozzi, and S. Valeri, "AFM nanoindentation: Tip shape and tip radius of curvature effect on the hardness measurement," J. Phys.: Condens. Matter 20(47), 474208 (2008).

${ }^{23}$ D. Torres-Torres, J. Muñoz-Saldaña, L. Gutierrez-Ladron-de Guevara, A. Hurtado-Macías, and M. Swain, "Geometry and bluntness tip effects on elastic-plastic behaviour during nanoindentation of fused silica: Experimental and FE simulation,” Modell. Simul. Mater. Sci. Eng. 18(7), 075006 (2010).

${ }^{24}$ S. Baek, S. K. Cho, and C. S. Seok, "A correction of geometric error of nano-indenter using atomic force microscope and finite element method," Key Eng. Mater. 321-323, 129-131 (2006).

${ }^{25} \mathrm{~F}$. Alisafaei, C.-S. Han, and N. Lakhera, "Characterization of indentation size effects in epoxy," Polymer Testing 40(0), 70-78 (2008).

${ }^{26} \mathrm{C}$. W. Shih, M. Yang, and J. C. M. Li, "Effect of tip radius on nanoindentation,” J. Mater. Res. 6(12), 2623-2628 (1991).

${ }^{27}$ B. Poon, D. Rittel, and G. Ravichandran, "An analysis of nanoindentation in linearly elastic solids," Int. J. Solids and Struct. 45(24), 6018-6033 (2008).

${ }^{28}$ M. Lucas, K. Gall, and E. Riedo, "Tip size effects on atomic force microscopy nanoindentation of a gold single crystal," J. Appl. Phys. 104(11), 113515 (2008).

${ }^{29}$ L. A. Berla, A. M. Allen, S. M. Han, and W. D. Nix, "A physically based model for indenter tip shape calibration for nanoindentation," J. Mater. Res. 25(04), 735-745 (2010).

${ }^{30} \mathrm{M}$. Troyon and L. Huang, "Correction factor for contact area in nanoindentation measurements," J. Mater. Res. 20(3), 610-617 (2005).

${ }^{31}$ J. Gong, H. Miao, and Z. Peng, "On the contact area for nanoindentation tests with Berkovich indenter: Case study on soda-lime glass," Mater. Lett. 58(7-8), 1349-1353 (2004).

${ }^{32}$ H. Bei, E. P. George, J. Hay, and G. M. Pharr, "Influence of indenter tip geometry on elastic deformation during nanoindentation," Phys. Rev. Lett. 95(4), 045501 (2005).

${ }^{33} \mathrm{~K}$. Xin and J. C. Lambropoulos, "Densification of fused silica: Effects on nanoindentation," in International Symposium on Optical Science and Technology (International Society for Optics and Photonics, 2000). 